\title{
Vancomycin-induzierte lineare IgA-Dermatose
}

\author{
Vancomycin-Induced Linear IgA Disease
}

Autoren

Institut
C. Leclerque, F. Ziller, T. Christoph, G. Schweigert, I. Feldmann-Böddeker

Hautklinik (Chefärztin Dr. med. I. Feldmann-Böddeker) DRK-Krankenhaus Chemnitz-Rabenstein

\section{Bibliografie}

DOI http://dx.doi.org/

10.1055/s-0030-1256662

Online-Publikation: 26. 7. 2011

Akt Dermatol 2011; 37:

366-368 ๑ Georg Thieme

Verlag KG Stuttgart · New York ISSN 0340-2541

\section{Korrespondenzadresse}

\section{Caterina Leclerque}

Hautklinik DRK-Krankenhaus

Chemnitz-Rabenstein

Unritzstraße 23

09117 Chemnitz

Leclerque.Caterina@

drk-chemnitz.de

\section{Zusammenfassung \\ $\nabla$}

Die lineare IgA-Dermatose (LAD) ist eine autoimmunologische vesikulo-bullöse Dermatose. Sie ist charakterisiert durch die lineare Ablagerung von IgA-Autoantikörpern entlang der dermo-epidermalen Junktionszone in der direkten Immunfluo-

\section{Einleitung}

Wir berichten über einen 75-jährigen Patienten, dem aufgrund einer mit MRSA infizierten Hüftprothese Vancomycin verabreicht wurde und der unter dieser Therapie eine lineare IgA-Dermatose entwickelte. Die lineare IgA-Dermatose ist zumeist eine idiopathisch auftretende Erkrankung. In seltenen Fällen kann diese Erkrankung auch durch Arzneimittel, am häufigsten durch Vancomycin, ausgelöst werden.

\section{Kasuistik \\ $\nabla$}

\section{Anamnese}

Ein 75-jähriger Mann wurde mit einer seit vier Tagen bestehenden bullösen Dermatose in unsere Klinik eingewiesen. Der Patient befand sich zuvor neun Wochen in stationärer orthopädischer Behandlung aufgrund einer infizierten Hüftprothese, die nach Explantation und Etappen-Lavagen durch die Re-Implantation einer Hüftgelenkprothese versorgt wurde. Während diesem stationären Aufenthalt in einer Klinik für Orthopädie hatte der Patient mehrfach antibiotische Therapien erhalten. Der Patient erhielt zunächst Cefuroxim über 15 Tage, folgend Vancomycin über 13 Tage wegen des Nachweises von multiresistentem Staphyloccocus epidermidis, über fünf Tage Cotrimoxazol bei Harnwegsinfekt, sowie später über vier Tage Metronidazol aufgrund einer Clostridien-Enterokolitis. Nachdem erneut eine Konta- reszenz. Viele Fälle verlaufen chronisch idiopathisch, in seltenen Fällen sind jedoch Medikamente, Infektionen und maligne Erkrankungen als ursächlich beschrieben. Wir berichten von einer linearen IgA-Dermatose eines 75-jährigen Patienten, die durch die Einnahme von Vancomycin induziert wurde.

mination mit einem multiresistenten Staphylococcus aureus nachgewiesen wurde, erhielt der Patient erneut Vancomycin i.v. nach Antibiogramm über sieben Tage. Unter der wiederholten Vancomycin-Gabe entwickelte der Patient am fünften Tag zunächst am Unterbauch und den unteren Extremitäten Blasen, sodass er in unsere Hautklinik verlegt wurde. An relevanten Nebendiagnosen bestanden vorwiegend kardiale Erkrankungen, wie chronische Herzinsuffizienz, Vorhofflimmern und arterielle Hypertonie, ferner eine Blutungsanämie und eine psychische Belastungsreaktion nach Wechsel einer Totalen Endoprothese des Hüftgelenks. Die entsprechende Hausmedikation bestand aus HCT, Ramipril, Nebivolol, Digitoxin, Pantoprazol und Melperon.

\section{Aufnahmebefund}

Zum Aufnahmezeitpunkt in unserer Klinik zeigte der Patient sowohl kokardenartige Erytheme mit zentraler Blasenbildung als auch pralle Blasen auf unauffälliger Haut am gesamten Integument mit Betonung des Abdomens, des unteren Rückens, der Oberschenkel ( $\bullet$ Abb.1), der Handflächen $(\bullet$ Abb. 2) und der Fußsohlen.

Des Weiteren bestanden nässende Erosionen an oben genannten Lokalisationen sowie im Genitalbereich und an der Mundschleimhaut. Es zeigte sich eine Rötung der Konjunktiva beidseits. Bei Aufnahme befand sich der Patient in deutlich reduziertem Allgemeinzustand. Die klinische Manifestation erweckte zunächst den Verdacht auf ein Stevens-Johnson-Syndrom (SJS). 


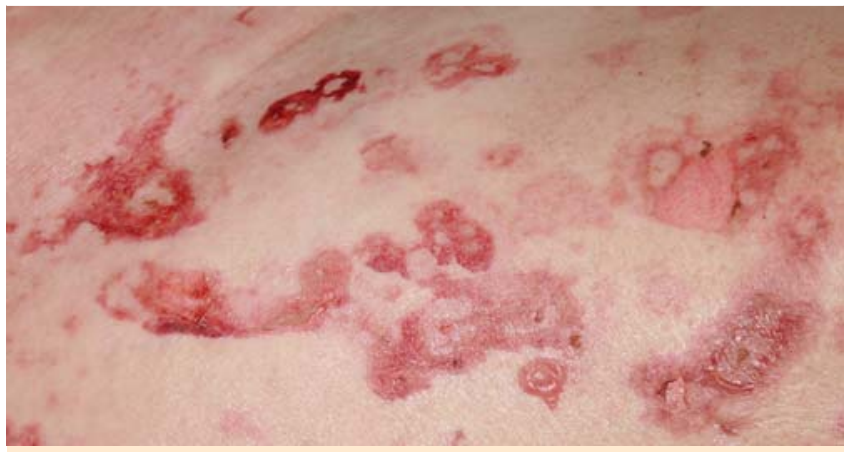

Abb. 1 Pralle und bereits rupturierte Blasen auf erythematöser oder normaler Haut (Oberschenkel).

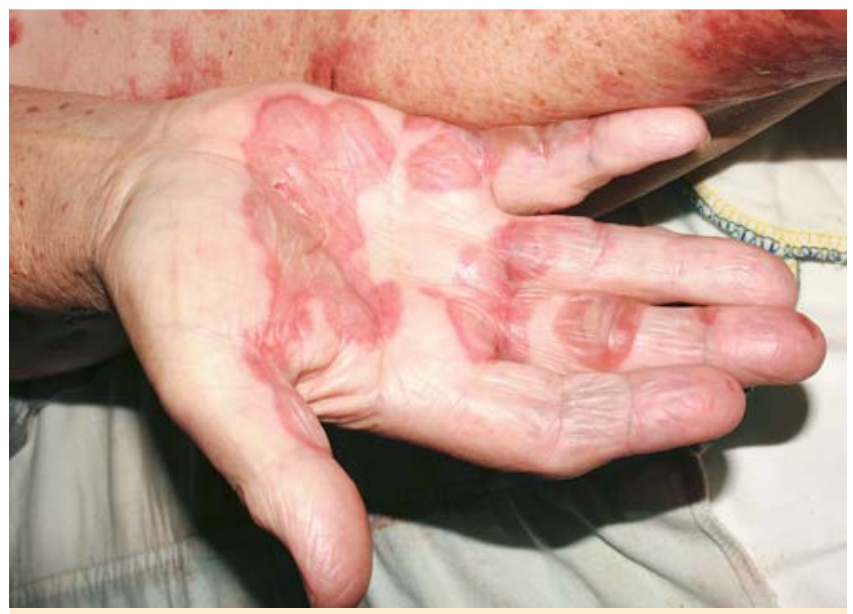

Abb. 2 Kokardenförmige Erytheme mit zentraler Blasenbildung an den Handflächen.

\section{Befunde diagnostischer Untersuchungen}

Zur weiteren Diagnostik erfolgte die Entnahme von Probebiopsien im Bereich der rechten Flanke. Die histologische Untersuchung zeigte eine subepidermale Blasenbildung ( $\boldsymbol{Q} \mathbf{A b b} . \mathbf{4})$ und imponierte lichtmikroskopisch aufgrund des Reichtums an eosinophilen Granulozyten wie ein bullöses Pemphigoid ( $\bullet$ Abb.5). Der Befund der direkten Immunfluoreszenz (DIF) zeigte eine kräftige Fluoreszenz in Form einer linearen Ablagerung von IgA im Bereich der Basalmembran. Dagegen fand sich keine Fluoreszenz in den Schnitten, die mit Antikörpern gegen IgG, IgM und C3 beschichtet worden waren. Ergänzend erfolgte die autoimmunologische Untersuchung mittels indirekter Immunfluoreszenz auf Affenösophagus, die keinen Nachweis zirkulierender IgG- oder IgA-Autoantikörper ergab. Ebenfalls wurde in der indirekten Immunfluoreszenz auf $\mathrm{NaCl}$-separierter humaner Spalthaut kein Nachweis zirkulierender IgG- oder IgA-Antikörper erbracht. Der Immunoblot mit konzentriertem Keratinozytenmedium zeigte zirkulierende IgA-Autoantikörper gegen die lösliche $120 \mathrm{kD}$ schwere Ektodomäne von BP180 (LAD-1). In der Zusammenschau der linearen Ablagerungen von IgA im Bereich der Basalmembran und der serologischen Befunde wurde die Diagnose einer linearen IgA-Dermatose gestellt.

\section{Therapie und Verlauf}

Vancomycin war bereits zwei Tage nach Auftreten der ersten Blasen abgesetzt worden. Es erfolgte initial eine hochdosierte Systemtherapie mit Kortikosteroiden, beginnend mit $250 \mathrm{mg}$ Prednisolon i.v., die folgend ausschleichend über drei Wochen

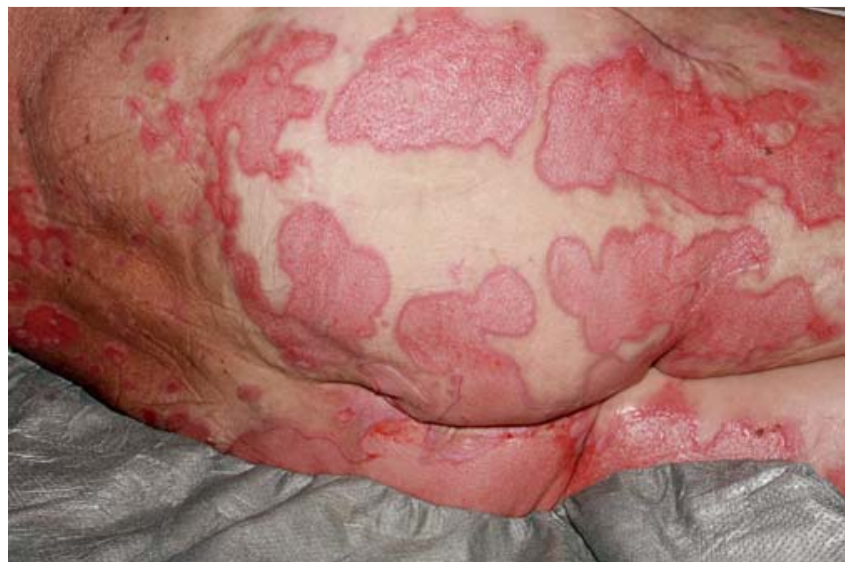

Abb.3 Im Verlauf Ausbildung großflächiger Erosionen gluteal, Lagerung auf Metallinefolie.

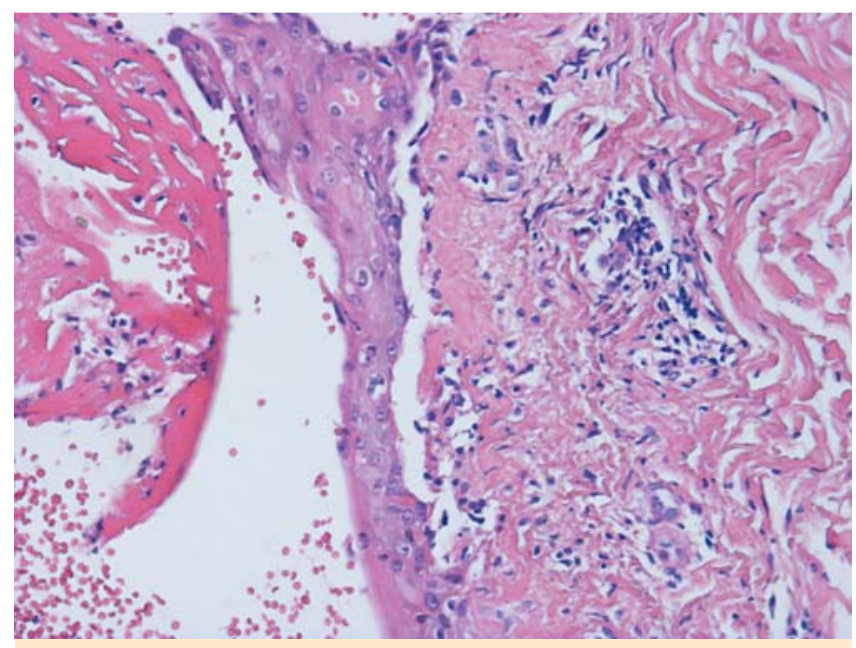

Abb. 4 Seitlicher Blasenrand mit subepidermaler Spaltbildung (HE-Färbung, $20 \times)$.

reduziert wurde. An Lokaltherapie verwendeten wir Triclosan $2 \%$ in DAC-Basiscreme bzw. Fusidinsäure $2 \%$ in DAC-Basiscreme an den Erosionen an Stamm und Extremitäten, gentamicinhaltige Creme an den mit Pseudomonas aeruginosa besiedelten Fußsohlen sowie Mundspülungen mit Krister-Lösung (Prednisolon $0,05 \%$, Propylenglycol $15 \%$, Tetracainhydrochlorid $1 \%$, Dexpanthenol 20\%, Kamillenextrakt EB6 4\% ad 100 g gereinigtes Wasser). Im Verlauf kam es nach Eröffnung der Blasen zu größflächigen Erosionen insbesondere am Rücken und gluteal, sodass der Patient auf Metallinefolie gelagert wurde $(\bullet$ Abb. 3 ).

Weiter erfolgte Volumensubstitution, Analgesie mit Metamizol und Tilidin/Naloxon oral sowie vor den Verbandswechseln zusätzlich mit Morphin s.c., Thromboseprophylaxe mit Fraxiparin und Infektionsprophylaxe mit Ciprofloxacin oral bei mikrobiologischem Nachweis von Pseudomonas aeroginosa im Hautabstrich der Erosionen an den Fußsohlen. Aufgrund der MRSA-Besiedlung erfolgte zudem eine standardmäßige Dekontamination durch tägliche antiseptische Ganzkörperwaschungen, Mundspülungen und Nasensalbe. Unter genannten Maßnahmen bildeten sich keine neuen Blasen, die Erosionen imponierten unter der oben genannten Therapie stets relativ sauber und zeigten sich nach etwa vier Wochen in deutlicher Abheilung.

Wegen wiederholter unterer gastrointestinaler Blutungen wurde der Patient nach vierwöchigem stationären Aufenthalt in eine 


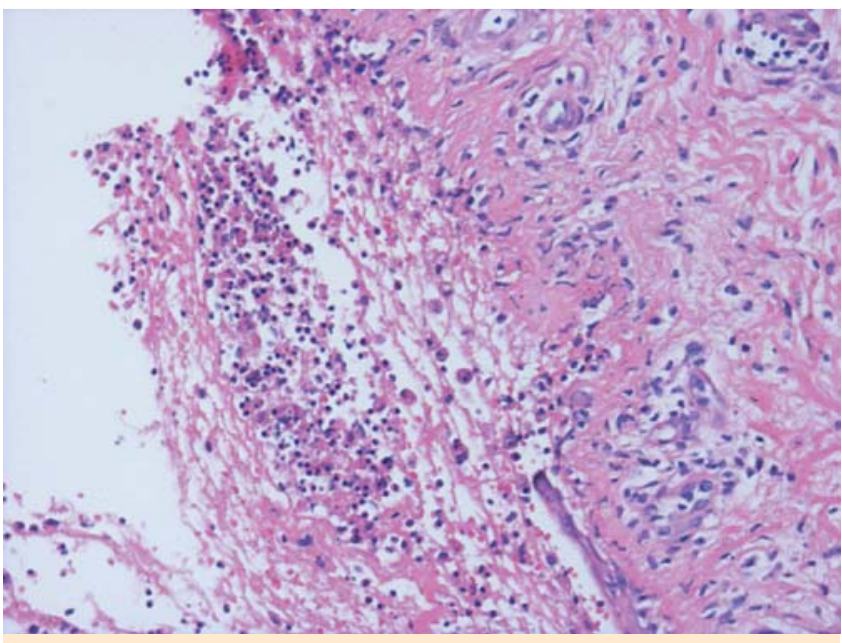

Abb. 5 Nachweis von Lymphozyten, neutrophilen und eosinophilen Granulozyten sowie Fibrin im Blasenbereich (HE-Färbung, $20 \times$ ).

Klinik für Chirurgie verlegt und es erfolgte eine Resektion eines blutenden Sigmadivertikels. Eine weitere Blutungsquelle konnte in der Gastro- und Koloskopie nicht eruiert werden. Im weiteren Verlauf kam es zur Abheilung des Hautbefundes.

\section{Diskussion}

$\nabla$

Die lineare IgA-Dermatose ist eine meist idiopathisch auftretende Autoimmunerkrankung. Eine seltene Variante stellt die durch Medikamente induzierte Form dar, die nach Absetzen der betreffenden Medikation selbstlimitierend verläuft. Vancomycin ist unter den angeschuldigten Medikamenten bisher am häufigsten beschrieben [1]. Genannt werden außerdem Penicillamin und Furosemid als Auslöser der LAD. Weiter liegen einzelne Fallberichte vor, in denen Captopril, Somatostatin, Rifampicin, Trimethoprim, Sulfamethoxazol, Phenytoin, Atorvastatin, Piroxicam, Lithium, Amiodaron, Cyclosporin, Cefamandol und Diclofenac als Auslöser einer LAD beschrieben werden [2]. Die Pathogenese der medikamentös induzierten LAD ist bisher noch nicht hinreichend erschlossen. Möglicherweise kann es durch oben genannte Medikamente zu einer Aktivierung von CD8+-T-Zellen im peripheren Blut und zu einer Erhöhung der Interleukin-5-Konzentration im betroffenen Gewebe kommen [3]. Es wird vermutet, dass Interleukin 5 und andere Zytokine die Expression von IgA beeinflussen [4]. In dem von uns beschriebenen Fall trat die Blasenbildung während der wiederholten Gabe von Vancomycin auf und sistierte drei Tage nach dem Absetzen der angeschuldigten Medikation. Eine Therapie mit Glukokortikoiden oder Dapson wird bei der medikamentös induzierten LAD im Allgemeinen nicht als notwendig erachtet [5], jedoch gaben wir von Beginn an systemisch Glukokortikoide unter der anfänglichen Vorstellung eines SJS.

Die LAD ist eine Erkrankung mit heterogenem klinischen Bild. Varianten der LAD mit dem klinischen Erscheinungsbild eines Erythema multiforme [6], eines SJS [5], einer toxischen epidermalen Nekrolyse (TEN) [7] und Fälle ohne Blasenbildung mit morbilliformem Exanthem [8] werden beschrieben. Der von uns vorgestellte Fall imponierte initial aufgrund der kokardenartigen Läsionen, der großflächigen Erosionen und der Beteiligung der Konjunktiva und der Mundschleimhaut als SJS. Eine Mundschleimhautbeteiligung bei LAD kann in $40-45 \%$ der Fälle auftreten [9]. Histologische Untersuchung und DIF sind aufgrund der polymorphen Klinik essenziell, um Differenzialdiagnosen wie bullöses Pemphigoid, Dermatitis herpetiformis Duhring, SJS, Erythema multiforme und TEN abzugrenzen und eine LAD zu identifizieren. Lineare Ablagerungen von IgA entlang der Basalmembran in der direkten Immunfluoreszenz sind das wichtigste Diagnosekriterium der LAD. Die direkte und indirekte Immunfluoreszenz ist bei SJS negativ [5]. Zirkulierende IgA-Autoantikörper sind bei medikamentös induzierter LAD häufig nicht nachweisbar [8], sodass die Diagnose auch in Abwesenheit zirkulierender Autoantikörper gestellt werden kann. Andererseits ist es durch die serologischen Techniken möglich, bereits durch den Nachweis der spezifischen Autoantikörper im Patientenserum die richtige Diagnose zu stellen [10].

\section{Abstract}

\section{Vancomycin-Induced Linear IgA Disease}

$\nabla$

Linear IgA disease (LAD) is an autoimmune bullous dermatosis. It is characterised by a linear band of $\operatorname{IgA}$ at the epidermal basement membrane on direct immunofluorescence. Although most reported cases are idiopathic, a subset of patients with LAD have been found to be drug-induced. We describe a case of LAD to Vancomycin in a 75 year old man.

\section{Literatur}

1 Montagnac $R$ et al. Drug induced linear IgA bullous dermatosis. Nephrologie 2003; 24 (6): 287-92

2 Benedicic Pilih A, Vizjak A et al. Drug induced linear IgA dermatosis. Acta Derm Venereol 2003; 12 (4):

3 Yawalkar $N$ et al. Drug-induced linear IgA bullous dermatosis associated with ceftriaxone- and metronidazole-specific T cells. Dermatology 1999; 199 (1): 25 - 30

4 Onodera $\mathrm{H}$ et al. Drug-induced linear IgA bullous dermatosis. J Dermatol 2005; 32 (9): 759-64

5 Jones DH, Todd M, Craig TJ. Early diagnosis is key in vancomycin-induced linear IgA bullous dermatosis and Stevens-Johnson syndrome. J Am Osteopath Assoc 2004; 104 (4): 157-63

6 Armstrong AW, Fazeli A, Yeh SW et al. Vancomycin-induced linear IgA disease manifesting as bullous erythema multiforme. J Cutan Pathol 2004; 31 (5): 393-397

7 Waldman MA, Black DR, Callen JP. Vancomycin-induced linear IgA bullous disease presenting as toxic epidermal necrolysis. Clin Exp Dermatol 2004; 29 (6): 633-636

8 Billet SE et al. A morbilliform variant of vancomycin-induced linear IgA bullous dermatosis. Arch Dermatol 2008; 144 (6): 774-778

9 Navi D, Michael DJ, Fazel N. Drug-induced linear IgA bullous dermatosis. Dermatol Online J 2006; 12 (5): 12

10 Rose E, Kasperkiewicz M, Rose C, Zillikens D. Bullöse Autoimmundermatosen - Neues zur Diagnostik und Therapie. Der Deutsche Dermatologe 2007; 9: 617-623 